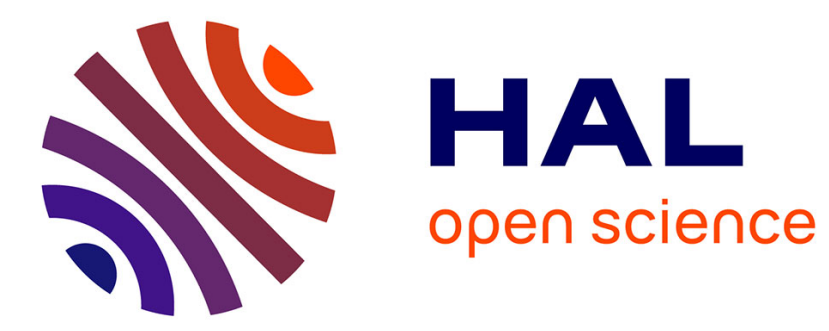

\title{
Lightweight Multitasking Support for Embedded Systems using the Phantom Serializing Compiler
}

Andre C. Nacul, Tony Givargis

\section{To cite this version:}

Andre C. Nacul, Tony Givargis. Lightweight Multitasking Support for Embedded Systems using the Phantom Serializing Compiler. DATE’05, Mar 2005, Munich, Germany. pp.742-747. hal-00181199

\author{
HAL Id: hal-00181199 \\ https://hal.science/hal-00181199
}

Submitted on 23 Oct 2007

HAL is a multi-disciplinary open access archive for the deposit and dissemination of scientific research documents, whether they are published or not. The documents may come from teaching and research institutions in France or abroad, or from public or private research centers.
L'archive ouverte pluridisciplinaire HAL, est destinée au dépôt et à la diffusion de documents scientifiques de niveau recherche, publiés ou non, émanant des établissements d'enseignement et de recherche français ou étrangers, des laboratoires publics ou privés. 


\title{
Lightweight Multitasking Support for Embedded Systems using the Phantom Serializing Compiler
}

\author{
André C. Nácul and Tony Givargis \\ Center for Embedded Computer Systems \\ Department of Computer Science \\ University of California, Irvine \\ \{nacul, givargis\}@ics.uci.edu
}

\begin{abstract}
Embedded software continues to play an ever increasing role in the design of complex embedded applications. In part, the elevated level of abstraction provided by a high-level programming paradigm immensely facilitates a short design cycle, fewer errors, portability, and reuse. Serializing compilers have been proposed as an alternative to traditional OS techniques, enabling a designer to develop multitasking applications without the need of OS support. In this work, we outline the inner workings of the Phantom serializing compiler and analyze the quality of the generated code with respect to memory and processing overheads. Our results show that such serializing compilers are extremely efficient, making them ideal to be used in design of highly parallel applications (e.g., multimedia, graphics, and signal processing applications).
\end{abstract}

\section{INTRODUCTION}

The functional complexity of embedded software continues to rise due to a number of factors such as sophisticated user interfaces, seamless operation across multiple communication protocols, need for security, and so on. Consequently, the development of embedded software poses a major design challenge. At the same time, the elevated level of abstraction provided by a high-level programming paradigm immensely facilitates a short design cycle, fewer errors, portability, and IP reuse. In particular, the concurrent programming paradigm is an ideal model of computation for design of embedded systems, which often encompass inherent concurrency.

Furthermore, embedded systems often have stringent performance requirements and, consequently, require a carefully selected and performance tuned embedded processor to meet specified design constraints. In recent years, a plethora of highly customized embedded processors have become available. As an example, Tensilica [11] provides a large family of highly customized applicationspecific embedded processors.

Such embedded processors ship with cross-compilers and the associated tool chain for application development. However, to support a multitasking application development environment, there is a need for an operating system (OS) layer that can support task creation, task synchronization, and task communication.

Such OS support is seldom available for each and every variant of the base embedded processor. In part, this is due to the lack of system memory and/or sufficient processor performance to afford the high performance penalty of having a full-fledged OS (e.g., in the case of micro-controllers such as the Microchip PIC [7] and the Philips 8051 [9]). Additionally, manually porting and verifying an OS to every embedded processor available is a high-cost job, in terms of time and money.

To fill the gap in realizing a multitasking application targeted at a particular embedded processor, researchers have proposed Phantom [8]. Phantom provides a fully automated source-to-source translator, taking a multitasking $\mathrm{C}$ program extended with POSIX as input and generating an equivalent, embedded processor independent, single-threaded ANSI C program, to be compiled using the embedded processor-specific tool chain. The output of Phantom is a highly tuned, correct (i.e., by construction) ANSI C program that embodies the application-specific embedded scheduler and dynamic multitasking infrastructure along with the user code.

In this work, we outline the inner workings of the Phantom serializing compiler. Specifically, we provide details on the architecture of Phantom generated code, such as memory layout, code organization, and the scheduler. Moreover, we analyze the quality of the Phantom generated code with respect to memory and processing overheads. Through our experiments, we show that such serializing compilers are extremely efficient, making them ideal to be used in design of highly parallel applications (e.g., multimedia, graphics, and signal processing applications).

Phantom is a new approach in addressing the challenge of multitasking support for embedded applications. We are unaware of any related work that use a similar compiler-based technique as that used in Phantom. However, we can identify three different approaches that address some of the design challenges solved with Phantom, namely a Virtual Machine (VM) based technique, template-based OS generation techniques, and static scheduling techniques. In the VM approach, portability is achieved, but with the overhead imposed by the VM layer. Moreover, the VM has to be ported to each new platform. To improve on these, solutions like JITs [1] and customized VMs for embedded platforms[13] have been proposed. In the template based OS generation, a custom OS is generated from a generic library of templates [4][5][12]. However, no single generic OS template can be used in the variety of embedded processors available. Finally, the static scheduling techniques [2][3][6] solve the static, a priori known, tasks class of problems, without addressing the dynamic multitasking issues.

The remainder of this work is organized as follows. In Section 2, we briefly describe Phantom, the source to source translator. In section 3, we discuss the architecture of the code generated by Phantom. In Section 4, we describe our experiments and give insight into the performance of code generated with Phantom. Finally, in Section 5, we state our conclusions.

\section{THE PHANTOM APPROACH}

\subsection{Introduction}

Input to Phantom is a multitasking program $P_{\text {input }}$, written in C. The multitasking is supported through the native Phantom API, 


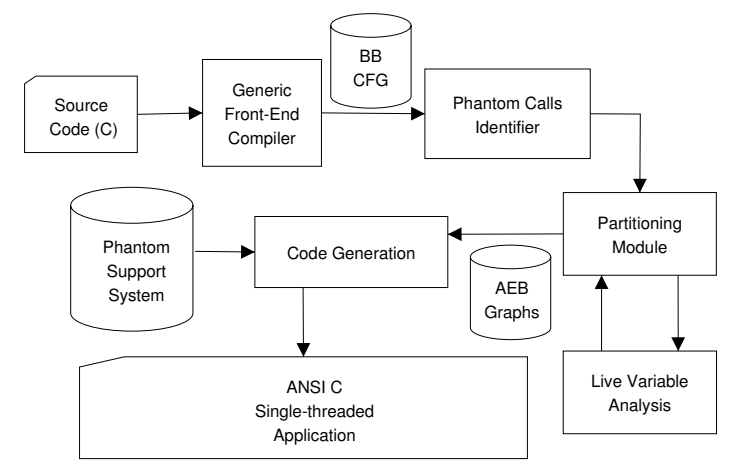

Figure 1: Phantom Compiler Architecture

which is a subset of the standard POSIX interface[10]. These primitives provide functions for task creation and management (e.g., task_create, task_join, etc.) as well as a set of synchronization variables (e.g., mutex_t, sema_t, etc.). Output of Phantom is a singlethreaded strict ANSI C program $P_{\text {output }}$ that is equivalent in functionality to $P_{\text {input }}$. More specifically, $P_{\text {output }}$ does not require any OS support and can be compiled by any ANSI C compiler into a self sufficient binary for a target embedded processor.

Figure 1 is the block diagram of Phantom. The multitasking $\mathrm{C}$ application is compiled with a generic front-end compiler to obtain the basic block (BB) control flow graph (CFG) representation. This intermediate $\mathrm{BB}$ representation is annotated, identifying Phantom primitives. The resulting structure is used by a partitioning module to generate non-preemptive blocks of code, which are called atomic execution blocks (AEBs), to be executed by the scheduler. Every task in the original code is partitioned into many AEBs, generating an AEB Graph. Then, a live variable analysis is performed on the AEB graphs and the result is fed back to the partitioning module to refine the partitions until acceptable preemption, timing, and latency are achieved. The resulting AEB graphs are then passed to the code generator to output the corresponding ANSI C code for each AEB node. In addition, the embedded scheduler, along with other $\mathrm{C}$ data structures and synchronization APIs are included from the Phantom system support library, resulting in the final ANSI C single-threaded code.

In the current version, Phantom is able to handle soft, firm, and event-driven real-time applications. All the modules pictured in Figure 1 are implemented and can be used in the automatic code generation process. Next, we briefly present the major components of Phantom. For a more complete description of these components, including discussions about code partitioning, please refer to [8].

\subsection{Scheduling and Synchronization}

We define the basic unit of execution, scheduled by the scheduler, an atomic execution block (AEB). An AEB is a block of code that is executed in its entirety prior to scheduling the next AEB. A task $T_{i}$ is partitioned into an AEB graph whose nodes are AEBs and edges represent control flow. Consider the example shown in Figure 2, implementing a game between two tasks that are picking up random numbers, and the corresponding CFG of function game of the example, pictured in Figure 3. Figure 3(a) shows the output of the compiler front-end that is fed to the partitioning module, annotated with the Phantom primitives. The partitioner adds two control basic blocks, setup and cleanup, as shown in Figure 3(b), and subsequently divides the function code into a number of AEBs,

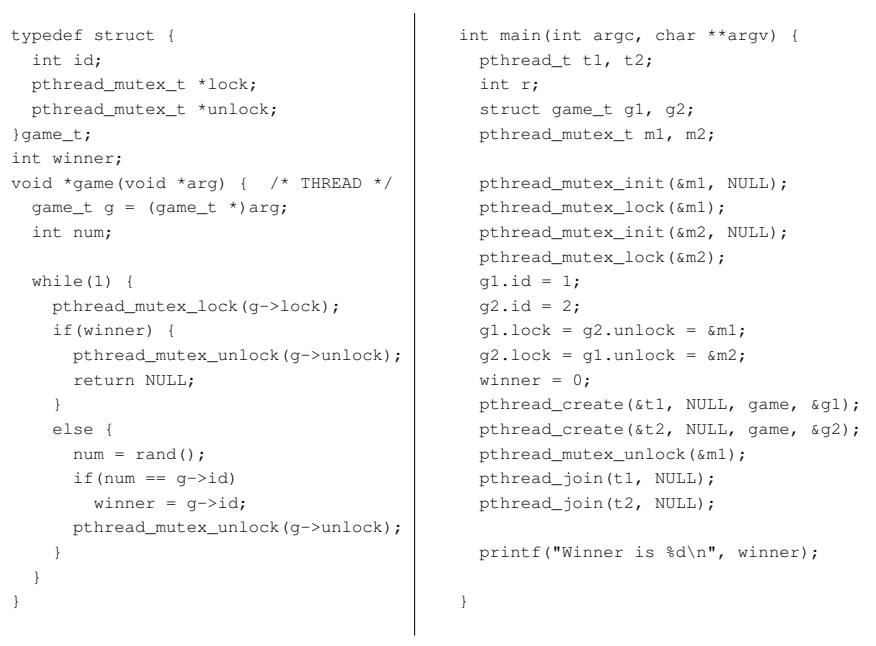

Figure 2: Code Example

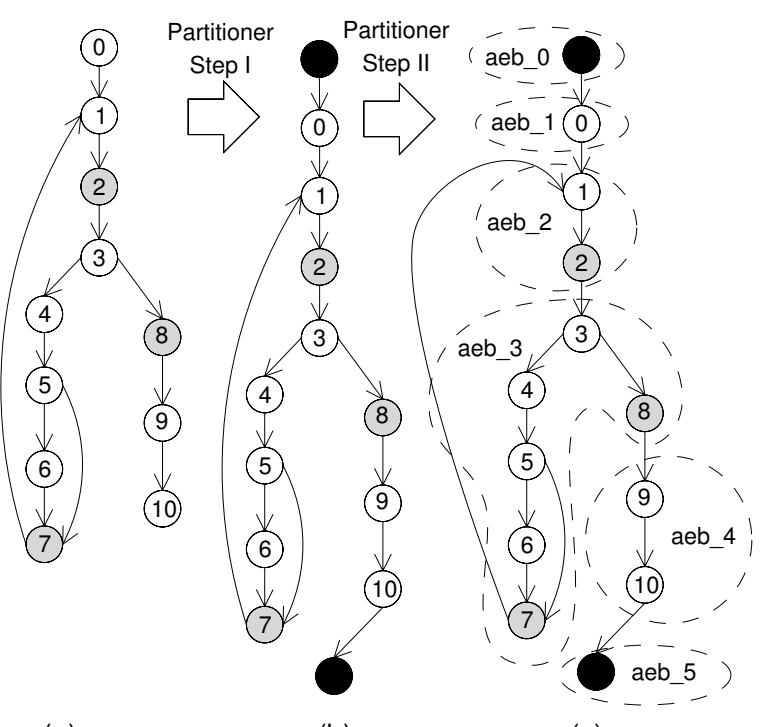

(a)

(b)

(c)

Figure 3: CFG Transformations for Function game

as shown in Figure 3(c), in a process we call phantomization.

Figure 3(c) shows the AEB graph of function game as being composed of AEBs $a e b \_0, a e b \_1, a e b \_2, a e b \_3, a e b \_4$ and $a e b \_5$. We note that an AEB node may be composed of one or more basic blocks. The termination of an AEB region transfers the control back to the scheduler. The scheduler, then, has a chance to activate the next AEB, from either the same task or from another task that is ready to run. A detailed description of the code layout and the scheduler implementation is in Section 3.

It may happen that a function $f$ in the original input code is phantomized (i.e., partitioned) into more than one AEB, each one of them being implemented as a separate region of code. In that case, there is a need for a mechanism to save the variables that are live on transition from one AEB to the other, so that the transfer of one AEB to another is transparent to the task. Also, every task must maintain its own copy of local variables during the execution of $f$ as part of its context. Phantom solves this issue by storing the values of local variables of $f$ in a structure inside the task context, 
emulating the concept of a function frame. The frame of a phantomized function $f$ is created in the $f_{\text {setup }}$ block, and cleaned up in the last AEB of $f$. These operations are included by the partitioner for every function that needs to be phantomized. They are represented by the dark nodes in Figure 3(b).

During runtime, there is a need to maintain, among others, a reference to the next AEB node that is to be executed some time in the future, called next_aeb, in the context information for each task that has been created. When a task is created, the context is allocated, the next_aeb field is initialized to the entry AEB of the task, and the task context is pushed onto the queue of existing tasks to be processed by the embedded scheduler.

The embedded scheduler is responsible for selecting and executing the next task, by activating the corresponding AEB of the task to be executed. The next_aeb reference of a task $T_{i}$ is used to resume the execution of $T_{i}$ by jumping to the region of code corresponding to the next AEB of $T_{i}$. At termination, every AEB updates the next_aeb of the currently running task to refer to the successor AEB according to the tasks's AEB Graph.

The scheduling algorithm in Phantom is a priority based scheme, as defined by POSIX. The way priorities are assigned to tasks, as they are created, can enforce alternate scheduling schemes, such as round-robin, in the case of all tasks having equal priority, or earliest deadline first (EDF), in the case of tasks having priority equal to the inverse of their deadline. Additionally, priorities can also be changed at run-time, so that scheduling algorithms based on dynamic priorities can be implemented.

Phantom implements the basic semaphore (sema_t in POSIX) synchronization primitive, upon which any other synchronization construct can be built. To implement semaphores, there is a need to add to a task $T_{i}$ 's context an additional field called status. Status is one of blocked or runnable and is set appropriately when a task is blocked waiting on a semaphore.

A semaphore operation, as well as a task creation and joining, is what is called a synchronization point. Synchronization points are identified by a gray node in Figure 3. At every synchronization point a modification in the state of at least one task in the system might happen. Either the current task is blocked, if a semaphore is not available, or a higher priority task is released on a semaphore signal, for example. Therefore, a function is always phantomized when synchronization points are encountered, and a call to a synchronization function is always the last statement in its AEB. At this point, the scheduler must regain control and remove the current task from execution in case it became blocked or is preempted by a higher priority task.

\subsection{Partitioning}

The partitioning of the code into AEBs is key to implementing multitasking at a high abstraction level. Recall that boundaries of AEB represent the points where tasks might be preempted or resumed for execution. Some partitions are unavoidable and must be performed for correctness, specifically, when a task invokes a synchronization operation, or when a task creates another task.

However, partitioning beyond what is needed for correctness impacts timing. In general, partitioning will determine the granularity level of the scheduling (i.e., the time quantum), as well as the task latency. A complete discussion on partitioning, with a deeper analysis of different partitioning schemes and algorithms for exploring different code partitions, is described in a separate work [8].

\subsection{Experiments with Phantom}

The Phantom approach has been successfully applied to a number of applications developed for testing the translation flow [8]. In

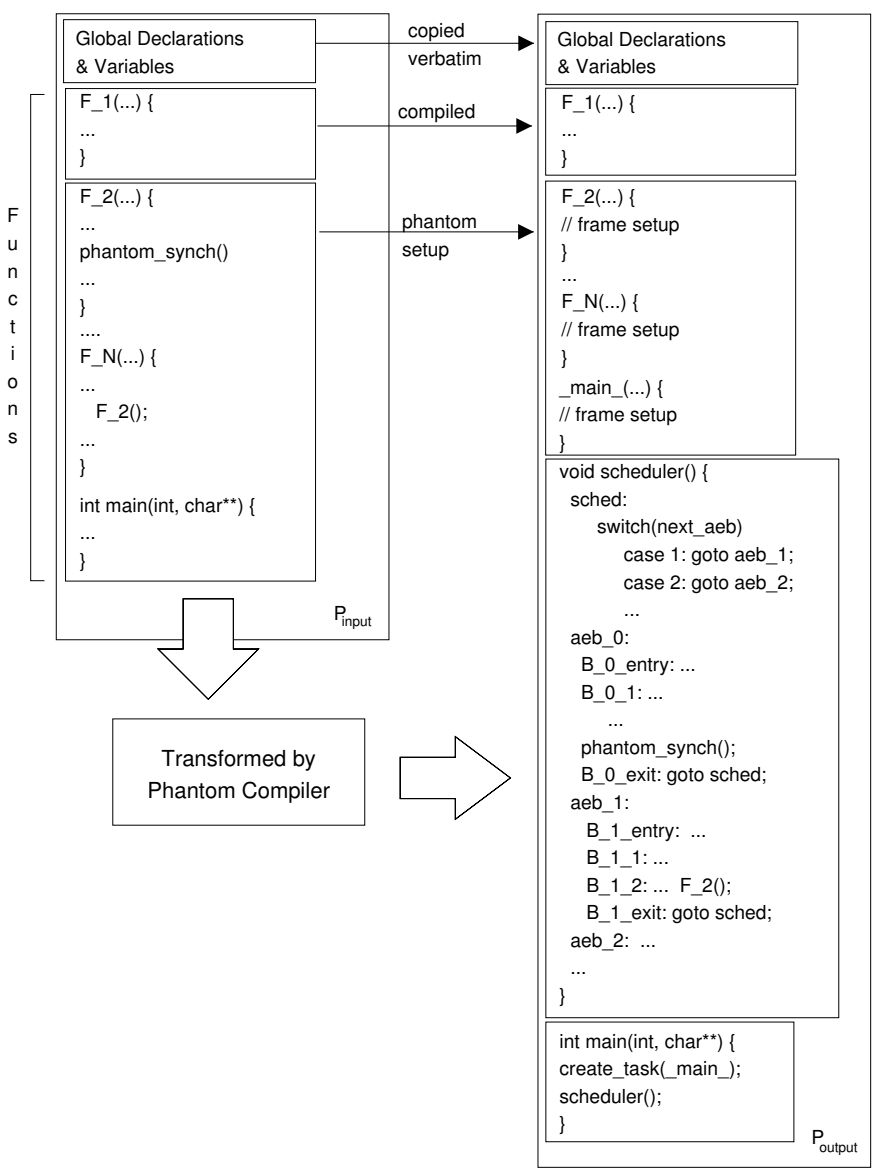

Figure 4: Code Layout of Input and Output Programs

summary, Phantom outperforms standard UNIX POSIX implementations, being 2 to 3 times faster in execution time [8]. In general, multitasking applications synthesized with Phantom show a much improved performance (i.e., low operating overhead). The reason is two fold. First, the generated application encompass a highly tuned multitasking framework that meets the application-specific needs. Second, the multitasking infrastructure itself is very compact and efficient, resulting in a much lighter overhead for context switching, task creation, and synchronization.

With regard to different partitioning schemes, it was shown that there is a clear correlation between partition and performance metrics, like latency and multitasking overhead [8]. Different code partitioning can reduce application latency by as much as two orders of magnitude, at the expense of an increase of the multitasking overhead by a factor of 120 [8]. The effect of partitioning is highly dependent on the application structure itself.

\section{GENERATED CODE ARCHITECTURE}

\subsection{Code Layout}

The code layout of the input program $P_{\text {input }}$, once processed by a $\mathrm{C}$ pre-processor, is conceptually organized in two sections, as shown in Figure 4. The first section contains all global declarations and variables, while the second section contains a set of functions. One of these functions is the main function, i.e., the entry point of the application. The Phantom output program $P_{\text {output }}$ is organized in five sections, as shown in Figure 4. The first sec- 
tion contains global declarations and variables. The second section contains a set of functions that are not phantomized. The third section contains a set of functions, each corresponding to one phantomized function of $P_{\text {input }}$. The fourth section contains a single function, called scheduler, which contains the code for all the phantomized functions, as well as the scheduling algorithm. Finally, the fifth section contains the main function of $P_{\text {output }}$.

All the functions of $P_{\text {input }}$ are analyzed and classified in two groups: the phantomized and non-phantomized functions. A function is phantomized if (i) it is the entry point of a task, (ii) contains a synchronization primitive(s), or (iii) calls a phantomized function. Note that, since main is the entry point of the first task that is created by default, it is automatically phantomized.

The second section of $P_{\text {output }}$ contains all non phantomized routines, copied over from $P_{\text {input }}$. The third section contains the setup functions, each corresponding to a phantomized function of $P_{\text {input }}$. A setup function is responsible for allocating the frame structure of each phantomized function. The frame and task context memory layout is described in a later subsection.

The next section of $P_{\text {output }}$ contains the phantomized functions, along with the scheduler. All of these (i.e., the phantomized functions and scheduler) are embodied into a single $\mathrm{C}$ function of $P_{\text {output }}$, namely scheduler. Recall that a phantomized function is partitioned into a set of AEBs, $a e b_{0}, a e b_{1}, \ldots, a e b_{n}$. An AEB $a e b_{i}$ is in turn composed of one or more basic blocks $B_{i, e n t e r}, B_{i, 2}, B_{i, 3}, \ldots, B_{i, \text { exit }}$. By definition, execution of AEB $a e b_{i}$ starts at the entry basic block of $B_{i, \text { enter }}$ and ends at the exit basic block $B_{i, e x i t}$. The exit basic block $B_{i, \text { exit }}$ of AEB aebi transfers control to a special basic block sched that serves as the entry point of the scheduling algorithm. The scheduler function contains all these basic blocks, starting with basic block sched, in low-level $\mathrm{C}$, using $\mathrm{C}$ labels to denote basic block boundaries and $\mathrm{C}$ goto statements as a branching mechanism. The scheduling algorithm is described in a later subsection.

Finally, the fifth section of $P_{\text {output }}$, contains an implementation of the main function, which creates a single task, corresponding to the main entry point of $P_{\text {input }}$, and calls the scheduler function to invoke the scheduling algorithm.

\subsection{Memory Layout}

Each time a task is created, memory is allocated to store its context. At any given time, a special global variable, named current, is made to point to the context of the running task by the scheduler. Moreover, a queue of running tasks, named tasks, is maintained, according to the priorities of each task, by the scheduler, as described in the following subsection. The context of a task is further defined in Figure 5.

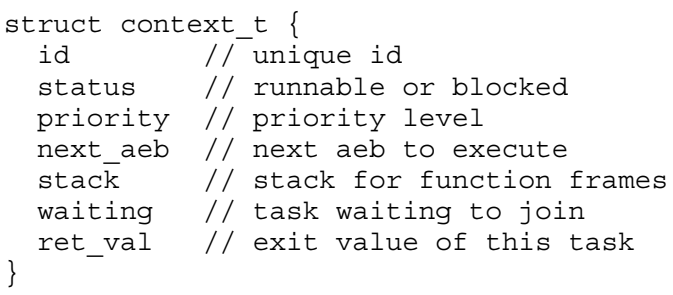

Figure 5: The Task Context Data Structure

Most of the fields of this structure were discussed earlier. Here, we focus on the stack field of a context. The purpose of the stack is to store the task-local data of each phantomized function. Moreover, the choice of a stack is to allow for recursion and nested function calls. The collection of all this data for a phantomized function $f$ is called $f$ 's frame, and is structured as shown in Figure 6. The frame of each phantomized function includes function arguments and local variables which are live at the boundary of its AEBs. The code in all basic blocks of $f$ 's AEBs access the most recent instance of $f$ 's frame.

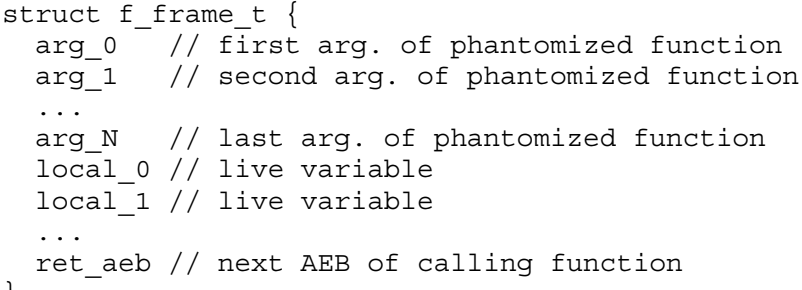

\section{Figure 6: The Frame Data Structure}

The stack is managed by the setup and the cleanup AEBs of phantomized functions. Specifically, when a function $g$ of the current task calls a phantomized function $f$, the setup function $f_{\text {setup }}$ is invoked. Then, $f_{\text {setup }}$ pushes $f$ 's frame onto the stack of the current task, copies $f$ 's arguments to the frame, saves the return AEB of the calling function $g$, and makes the current task's next AEB point to the entry AEB of $f$. The structure of the setup function is shown in Figure 7.

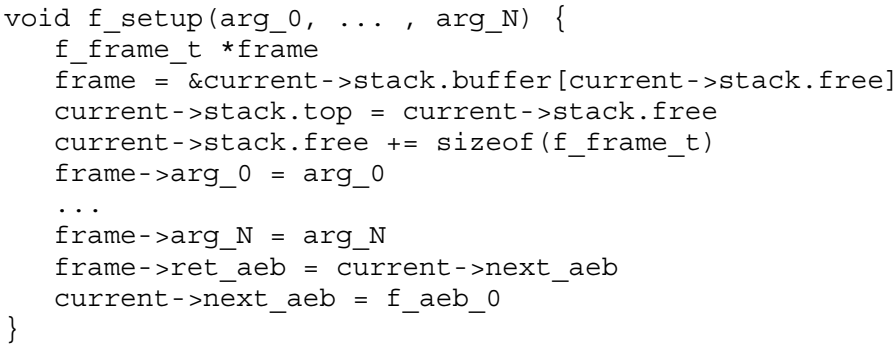

\section{Figure 7: Code Structure of Setup Functions}

Conversely, when a called function $f$ complete its execution, the cleanup AEB aeb exit of $f$ performs the following. First, it restore the current task's next AEB to point to the next AEB of the calling function $g$, which was stored in the frame of $f$ by the $f$ 's setup function. Then, it pops the frame of the current task's stack, as shown in Figure 8.

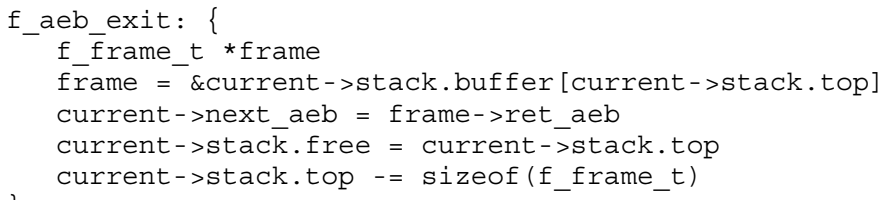

Figure 8: Code Structure of Cleanup AEB

\subsection{Scheduler}

The scheduler's code is included in the same $\mathrm{C}$ function containing the phantomized functions, called scheduler. The scheduling algorithm makes use of a priority queue that stores all the runnable tasks. The priority queue guarantees that the highest priority task is always the first task in the queue. In case of a priority tie among two or more tasks, the scheduler implements a round-robin scheme among them, so that all equal-priority tasks fairly share the 
processor. When a task is selected by the scheduler for execution, the global current pointer is updated accordingly.

Each AEB returns the execution to the scheduler upon termination. This is accomplished through a jump to the first basic block of the scheduler. Once the scheduler determines the next task $T_{i}$ to be executed, it uses $T_{i}$ 's next_aeb reference to transfer control back to the next AEB. The transfer of control from the scheduler to the next AEB of the running task is implemented using a switch statement containing goto's to all AEB's of the application. When the AEB completes execution, control is returned to the scheduler, which then pushes the current task's context back to the queue of runnable tasks if the task is not blocked or terminated. An overview of the scheduler is depicted in Figure 9.

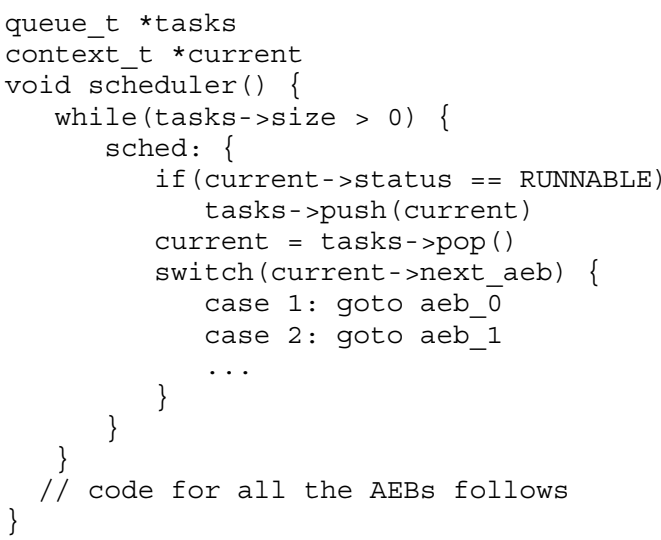

\section{Figure 9: Code Structure of Scheduling Function}

An optimization in the scheduling algorithm allows a task to execute more than one AEB each time it is selected from the priority queue. We call this a short context switch. With the short context switch, it is possible to save the overhead of pushing/popping a new task from the priority queue with a bypass. A full context switch is executed every so often, alternating short and full context switches with a pre-determined ratio. A full context switch ensures a fair sharing of the processor among equal-priority tasks.

In order to implement the short context switch, we add a counter to the scheduling algorithm, used to keep track of the number of consecutive short context switches performed. The counter is initialized to a value representing the ratio between short and full context switches. The value of the counter defines a time quantum, i.e., a number of consecutives AEBs of the same task to be executed before a full context switch. The counter is decremented at every short context switch, and a full context switch is executed once the counter reaches zero and expires. Obviously, a full context switch can happen before the counter expires, in the case that a task is blocked or terminates. Alternatively, a timer can be used in place of a counter, yielding a real time-sharing of the processor in the round-robin approach. Figure 10 shows the optimized scheduler algorithm, incorporating the short context switch optimization.

In Phantom, for efficiency reasons, a limited priority queue is implemented. A limited priority queue is one that allows a finite, and a priori known, number of priority levels (e.g., 32). However, this does not pose any limitations, since the number of priority levels, required by the application, can be provided to the Phantom serializing compiler. The implementation of the priority queue is as follows. A separate array-based queue is allocated for each priority level, which are accessed by the scheduler in order of highest to lowest priority. Manipulation of the array-based queues at each priority level is very efficient, and takes constant time. At any given

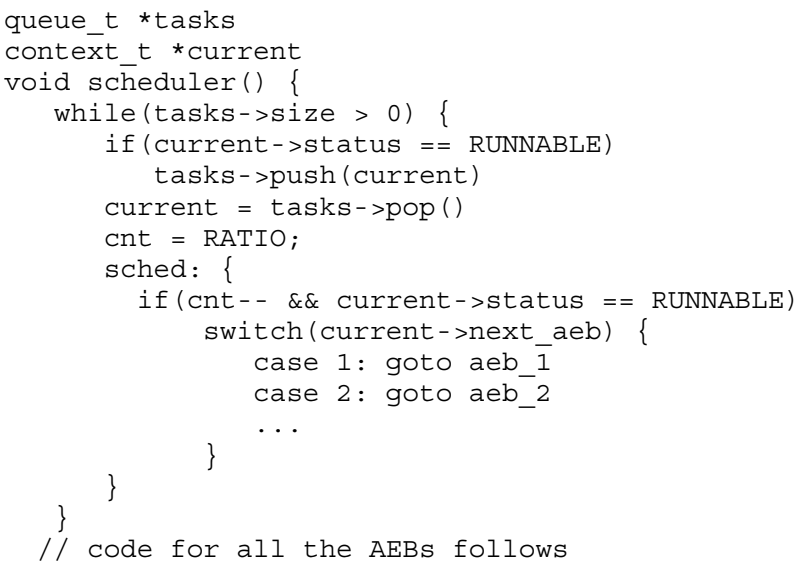

Figure 10: Code Structure of Optimized Scheduling Function

point, a reference is maintained to the highest non-empty priority queue. Given this, the overall access to the queue of runnable tasks by the scheduler requires constant running time, regardless of the number of runnable tasks.

\section{EXPERIMENTAL RESULTS}

A set of synthetic benchmarks was implemented to evaluate the overhead imposed by the Phantom multitasking infrastructure. Various parameters of Phantom were evaluated, like context switching overhead, task creation cost, task joining cost, and mutex synchronization cost.

Cost was measured as the average number of instructions executed on the host processor for performing a particular operation (e.g., task creation, task joining, etc.) We compiled and executed the applications on a UltraSPARC-IIe workstation, $500 \mathrm{Mhz}$, with $256 \mathrm{Mb}$ of RAM running Solaris operating system. We used cputrack tool to obtain number of instructions and CPU cycles executed by a target program. (cputrack uses hardware counters to track CPU usage). All benchmarks were compiled with GCC v3.3. The time cost of each metric was calculated from the average CPI (cycles per instruction) of each benchmark, associated with the processor cycle time.

For each benchmark, designed to measure a particular metric, we first obtained a baseline execution count. The baseline execution count accounted for all the computation code less the Phantom generated multitasking infrastructure. Then, the multitasking infrastructure was enabled and instruction counts were re-evaluated. The difference between the baseline and the version with the multitasking infrastructure gave us a measure of the performance of Phantom for that metric. All experiments in this phase were performed using at most one task active and a single priority level On average, Phantom multitasking infrastructure overhead is small, and has an impact of less than $1 \%$ in the execution time of the synthetic benchmarks. Our results are summarized in Table 1.

Next, we evaluated the impact of multiple task and multiple priorities in task context switch. In these experiments, we used a mixed scheduler, with a 10:1 ratio between short and full context switch. Figure 11 shows the results. Here, the horizontal axis of the plot depicts the number of runnable tasks in the system (i.e., one of $2,10,20,50,100,500$, and 1000 tasks). The vertical axis of the plot depicts the average number of instructions for performing a context switch.

We note from Figure 11 that the overhead of context switch is 
Table 1: Phantom Multitasking Performance Results

\begin{tabular}{|l|r|r|r|r|}
\hline \multirow{2}{*}{ Metric } & \multicolumn{2}{|c|}{ No optimization $(-\mathrm{O} 0)$} & \multicolumn{2}{|c|}{ With optimization $(-\mathrm{O} 2)$} \\
\cline { 2 - 5 } & Instructions & Time $(\mu \mathrm{s})$ & Instructions & Time $(\mu \mathrm{s})$ \\
\hline \hline full context switch & 427 & 1.81 & 206 & 0.47 \\
short context switch & 82 & 0.35 & 37 & 0.08 \\
mixed context switch (10:1) & 124 & 0.52 & 58 & 0.13 \\
task creation & 1113 & 4.74 & 833 & 1.90 \\
task join & 506 & 2.15 & 227 & 0.52 \\
mutex lock & 68 & 0.29 & 40 & 0.09 \\
\hline
\end{tabular}

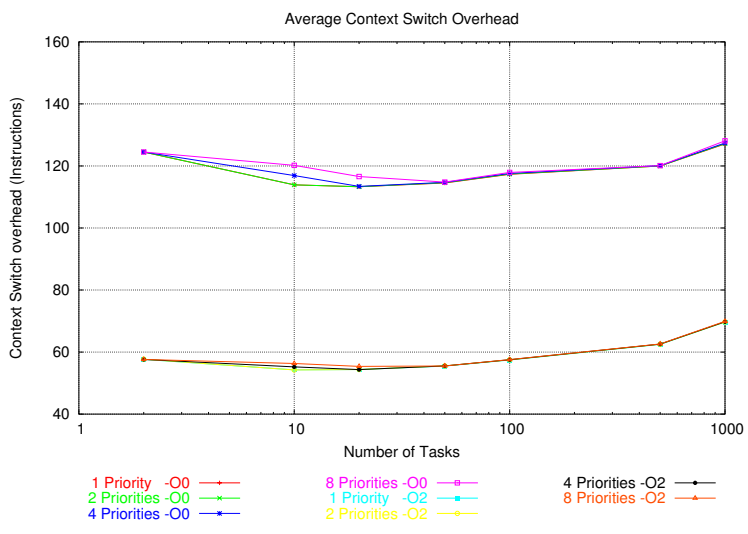

Figure 11: Phantom Context Switch Cost in Instructions with Multiple Threads and Multiple Priorities

small, fairly constant, and independent of the number of runnable tasks in the system. A similar result was obtained for task creation overhead. Contrary to intuition, there is initially a slight decrease in the context switch time when the number of tasks increase. With a small number of tasks, there are more reorganizations in the priority queue, since every context switch can possibly insert a task with a different priority in the queue. As the number of tasks increase, reorderings are less constant, since a task with the same priority is likely to be in the queue already. Therefore, context switch is slightly faster. Nevertheless, the impact of Phantom in the execution time of the benchmarks is typically less than $1 \%$, for the applications tested. A similar trend is observed with respect to the number of priorities, i.e., increasing the number of priorities does not have a significant impact on context switch time. As before, there is a slight difference in context switch time when few tasks are present. In this case, the priority queue has to be reorganized more often, increasing the context switch by a small margin. The efficiency of the Phantom generated code makes it practical for designing multimedia, digital signal processing, or other highly parallel applications, using the concurrent programming model.

\section{CONCLUSIONS}

In this work, we have outlined the code architecture and memory layout of Phantom serializing compiler. A serializing compiler is a source-to-source translator that takes a POSIX compliant multitasking $\mathrm{C}$ program as input and generates an equivalent, embedded processor independent, single-threaded ANSI C program, to be compiled using the embedded processor-specific tool chain. Serializing compilers have been proposed as an alternative solution, enabling a designer to develop multitasking applications without the need of OS support. We have also analyzed the quality of the generated code with respect to memory and processing overheads. Our results show that such serializing compilers are extremely efficient, making them ideal to be used in design of highly parallel applications (e.g., multimedia, graphics, and DSP applications).

Our future direction of research is to incorporate real-time scheduling into the Phantom serializing compiler. Specifically, we plan to allow deadline driven task scheduling using dynamic priorities. In addition, we would like to investigate power management strategies (e.g, voltage scaling, power modes, memory management, etc.) to be automatically generated by the Phantom serializing compiler.

\section{Acknowledgment}

This work was supported by the National Science Foundation award number CCR-0205712 and by CAPES Foundation, Brazil, award number 1054/01-5.

\section{REFERENCES}

[1] J. Aycock. A Brief History of Just-In-Time. ACM Computing Surveys, 35(2):97-113, Jun. 2003.

[2] J. Cortadella et. al. Task Generation and Compile-Time Scheduling for Mixed Data-Control Embedded Software. In Proc. of DAC, Jun. 2000.

[3] S. Edwards. Tutorial: Compiling Concurrent Languages for Sequential Processors. ACM Trans. on Design Automation of Electronic Systems, 8(2):141-187, Apr. 2003.

[4] L. Gauthier, S. Yoo, and A. Jerraya. Automatic Generation and Targeting of Application-Specific Operating Systems and Embedded Systems Software. IEEE Trans. on Computer-Aided Design of Integrated Circuits and Systems, 20(11):1293-1301, Nov. 2001.

[5] A. Gerstlauer, H. Yu, and D. Gajski. RTOS Modeling for System Level Design. In Proc. of DATE, Mar. 2003.

[6] B. Lin. Efficient Compilation of Process-Based Concurrent Programs without Run-Time Scheduling. In Proc. of DATE, Feb. 1998

[7] Microchip Inc. http://www.microchip.com.

[8] A. Nacul and T. Givargis. Code Partitioning for Synthesis of Embedded Applications with Phantom. In Proc. of ICCAD, Nov. 2004

[9] Phillips Inc. http://www.philips.com.

[10] POSIX Open Group. http://www.opengroup.org.

[11] Tensilica Inc. http://www.tensilica.com.

[12] S. Vercauteren, B. Lin, and H. D. Man. A Strategy for Real-Time Kernel Support in Application-Specific HW/SW Embedded Architectures. In Proc. of DAC, Jun. 1996.

[13] V. Verdiere, S. Cros, C. Fabre, R. Guider, and S. Yovine. Speedup Prediction for Selective Compilation of Embedded Java Programs. In Proc. of EMSOFT, Oct. 2002. 